Preprint typeset in JINST style - HYPER VERSION

\title{
Neutron calibration facility with an Am-Be source for pulse shape discrimination measurement of CsI(TI) crystals
}

\author{
H.S. Lee ${ }^{a}{ }^{*}$ H. Bhang ${ }^{b}$, J.H. Choi ${ }^{b}$, S. Choi ${ }^{b}$, I.S. Hahn ${ }^{c}$, E.J. Jeon ${ }^{d}$, H.W. Joo ${ }^{b}$, \\ W.G. Kang ${ }^{d}$, G.B. $\mathbf{K i m}^{b, d}$, H.J. $\mathbf{K i m}^{e}$, K.W. $\mathbf{K i m}^{b}$, S.C. $\mathbf{K i m}^{b}$, S.K. Kim ${ }^{b}$, Y.D. $\mathbf{K i m}^{d, f}$, \\ Y.H. Kim ${ }^{d, g}$, J.H. Lee ${ }^{b}$, J.K. Lee ${ }^{b}$, D.S. Leonard ${ }^{h}$, J. Li ${ }^{d}$, S.S. Myung ${ }^{b}$, S.L. Olsen ${ }^{b}$, and \\ J.H. So ${ }^{d}$ \\ ${ }^{a}$ Department of Physics, Ewha Womans University, Seoul 120-750, Korea \\ ${ }^{b}$ Department of Physics and Astronomy, Seoul National University, Seoul 151-747, Korea \\ ${ }^{c}$ Department of Science Education, Ewha Womans University, Seoul 120-750, Korea \\ ${ }^{d}$ Center for Underground Physics, Institute for Basic Science (IBS), Daejon 305-811, Korea \\ ${ }^{e}$ Department of Physics, Kyungpook National University, Daegu 702-701, Korea \\ ${ }^{f}$ Department of Physics, Sejong University, Seoul 143-747, Korea \\ ${ }^{g}$ Korea Research Institute of Standards and Science, Daejon 205-340, Korea \\ ${ }^{h}$ Department of Physics, The University of Seoul, Seoul 130-743, Korea \\ E-mail: hyunsuleedewha.ac.kr
}

\begin{abstract}
We constructed a neutron calibration facility based on a 300-mCi Am-Be source in conjunction with a search for weakly interacting massive particle candidates for dark matter. The facility is used to study the response of $\mathrm{CsI}(\mathrm{Tl})$ crystals to nuclear recoils induced by neutrons from the Am-Be source and comparing them with the response to electron recoils produced by Compton scattering of $662-\mathrm{keV} \gamma$-rays from a ${ }^{137} \mathrm{Cs}$ source. The measured results on pulse shape discrimination (PSD) between nuclear- and electron-recoil events are quantified in terms of quality factors. A comparison with our previous result from a neutron generator demonstrate the feasibility of performing calibrations of PSD measurements using neutrons from a Am-Be source.
\end{abstract}

KEYWORDS: CsI(Tl) crystal; Dark matter; WIMP; PSD; Neutron source; Quality factor.

\footnotetext{
${ }^{*}$ Corresponding author.
} 


\section{Contents}

1. Introduction 1

2. Experiments 2

3. Analysis

3.1 Neutron identification

$3.2 \mathrm{CsI}(\mathrm{Tl})$ data analysis

3.3 Pulse shape discrimination 5

4. Conclusions 8

\section{Introduction}

It is well known that the largest component of the gravitationally interacting matter in the Universe is not ordinary matter but nonbaryonic exotic dark matter. Weakly Interacting Massive Particles (WIMPs) are strong candidates for this dark matter [1, 2]. WIMPs are expected to interact with ordinary matter via elastic scattering from nuclei and could be directly detected by measuring the recoil energy of the nuclei that are expected to be in the $<100 \mathrm{keV}$ [3] energy range. Because of the high stopping power of recoil nuclei with these energies, the light yield of a scintillation detector is typically a factor of 5-10 times lower than that produced by $\gamma$-rays of equivalent energy. This reduction in light output is parameterized by a quantity called the quenching factor. As a result of this, WIMP interactions in typical scintillation detectors produce signals with visible energies of only a few keV.

Due to the effects discussed above, it is crucial for WIMP search experiments to have a quantitative understanding of the detector response to low-energy recoiling target nuclei. It is also important to be able to reduce the environmental backgrounds that are mostly due to $\gamma$ and $\beta$ decays by exploiting differences in the detector responses to nuclear- and orbital-electron-recoil-induced signals in the detector. The high stopping power of a recoil nucleus produces a high density of ionization that results in a time profile for photoelectron production that is different from that produced by electron recoils [\#]. This characteristic property makes particle identification possible using the time structure of the signal pulse. The pulse shape discrimination (PSD) capabilities of various scintillating counters have been previously reported [5, 6, 7, 8, 9, 10, 11].

Among various scintillating crystals, $\mathrm{CsI}(\mathrm{Tl})$ has special merits owing to its relatively large light yields and good PSD capability [7, 12]. Also, large volume CsI(Tl) crystals are relatively easy to fabricate, in part because they are not very hygroscopic. The large atomic masses of Cs and I nuclei enhance the cross section for coherent WIMP-nucleus spin-independent interactions (which depend on $A^{2}$ ). Also, Cs and I nuclei have relatively large spin factors for protons $\left(S_{p}\right)$ that 
enhance the cross section for WIMP-proton spin-dependent interactions (which depend on $S_{p}^{2}$ ). Several studies aimed at measuring the PSD capability of CsI(Tl) crystals have been performed with neutron beams generated by neutron generators [7, 13] and a ${ }^{252} \mathrm{Cf}$ neutron source [8]. These studies demonstrated the good PSD capability of CsI(Tl) crystals in comparison with those of $\mathrm{NaI}(\mathrm{Tl})$ crystals.

Previous studies of the PSD capability of $\mathrm{CsI}(\mathrm{Tl})$ crystals in our group used mono-energetic neutrons produced by the ${ }^{3} \mathrm{H}(\mathrm{p}, \mathrm{n})^{3} \mathrm{He}$ reaction of a neutron generator at the Korea Institute of Geoscience and Mineral resources (KIGAM) [14]. Even though this mono-energetic neutron beam facilitates the understanding of the deposited energy in the crystal so that one can measure quenching factors, the high costs of construction and maintenance make them impractical for a universitybased research facility. Our opportunities to use neutron beam generators at remote sites were limited by available beam time and funding.

For WIMP searches, however, we need to understand the dependence of the PSD capability for each detector on many variables, such as the raw powder used for growing the crystal, the growth method, the company that grew the crystal, the photon sensor, and the electronic readout, including the trigger and data-acquisition system. In order to measure the PSD power of the CsI(Tl) crystals used for our searches within time and cost constraints, we constructed a neutron calibration facility based on a recycled 300-mCi Am-Be neutron source. There was another study that used a neutron source $\left({ }^{252} \mathrm{Cf}\right)$ for the PSD measurement of the CsI crystal [8]. That measurement demonstrated the feasibility of the use of a neutron source for the PSD measurement. Our facility has a special design that includes $\gamma$ and neutron tagging detectors that can reduce possible contamination of the electron recoil events caused by environmental background as well as accompanying $\gamma \mathrm{s}$ of the neutron source in neutron calibration data. We measured the responses to nuclear recoils induced by neutrons from this source and compared them with the responses to electron recoils produced by a ${ }^{137} \mathrm{Cs} \gamma$ source. The performance of this Am-Be neutron calibration facility for the PSD measurement was found to be compatible with that using the neutron generator.

\section{Experiments}

We constructed a neutron calibration facility at Seoul National University using a 300-mCi Am-Be source recycled from Korea Cancer Center Hospital. Alpha particles emitted by ${ }^{241}$ Am impinge on a ${ }^{9} \mathrm{Be}$ target and produce the $(\alpha, n)$ reactions:

$$
\begin{aligned}
& \alpha+{ }^{9} \mathrm{Be} \rightarrow{ }^{12} \mathrm{C}+\mathrm{n}(\sim 50 \%), \\
& \alpha+{ }^{9} \mathrm{Be} \rightarrow{ }^{12} \mathrm{C}^{*}+\mathrm{n}(\sim 50 \%), \\
& { }^{12} \mathrm{C}^{*} \rightarrow{ }^{12} \mathrm{C}+\gamma(4.4 \mathrm{MeV}) .
\end{aligned}
$$

These neutrons were used to irradiate $\mathrm{CsI}(\mathrm{Tl})$ crystals with the arrangement depicted in Fig. 1. The neutron source is surrounded by liquid scintillator (LSC) composed of 95\% mineral oil and $5 \%$ pseudocumene with a collimated aperture in the $\mathrm{CsI}(\mathrm{Tl})$ crystal direction. The LSC acts as a tagging detector for the 4.4-MeV $\gamma$ particles as well as a passive neutron shield. Additional passive shielding for reducing uncollimated neutron and $\gamma$ fluxes to a level sufficient for safe operation, is provided by a structure comprised of $5 \mathrm{~cm}$ of lead and $10 \mathrm{~cm}$ of polyethylene. The CsI(Tl) 


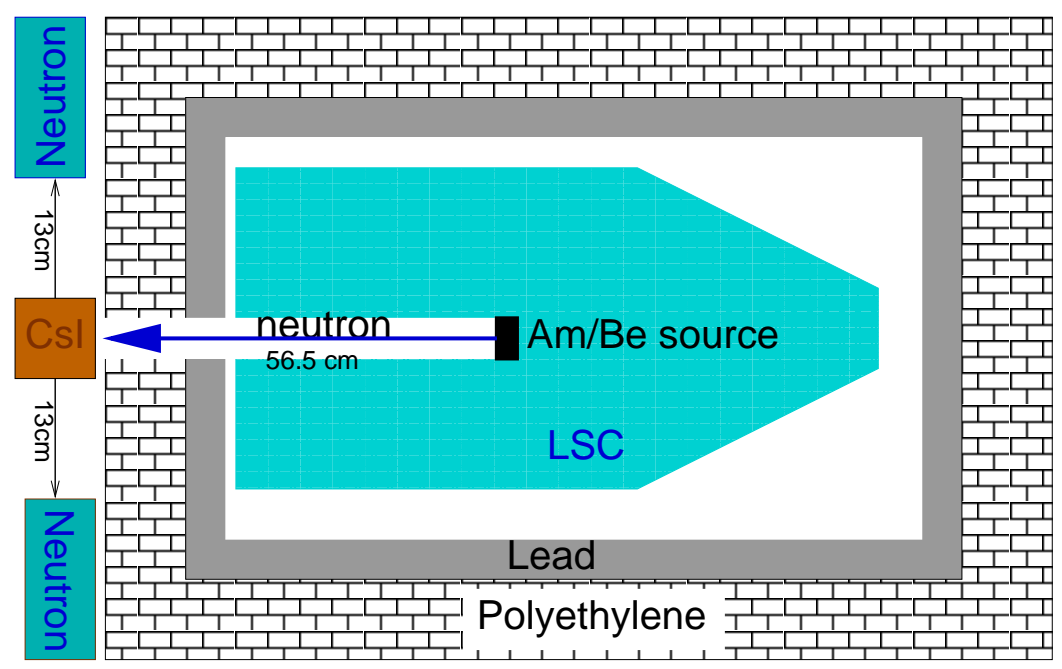

Figure 1. Neutron calibration setup.

crystal under study is located at the exit of the collimated aperture as shown in the figure. To identify neutrons scattered from CsI, two neutron tagging detectors are located at $90^{\circ}$ from the line between the neutron source and the $\mathrm{CsI}(\mathrm{Tl})$ crystal. The neutron detectors consist of BC501A liquid scintillator contained in cylindrical $0.5 \ell$ stainless-steel containers. Each neutron detector is read-out by a single, 2-inch photomultiplier tube (PMT) (H1161, Hamamatsu Photonics) and surrounded by $10-\mathrm{cm}$ paraffin and 5 -cm lead blocks to reduce external background.

We used two different $\mathrm{CsI}(\mathrm{Tl})$ crystals from two different crystal-growing companies: a $3 \times$ $3 \times 3 \mathrm{~cm}^{3}$ crystal from Beijing Hamamatsu (Beijing crystal) and a $3 \times 3 \times 2 \mathrm{~cm}^{3}$ crystal from SCICCA in Shanghai (Shanghai crystal). These two crystal companies grew full-sized $\left(8 \times 8 \times 30 \mathrm{~cm}^{3}\right)$ $\mathrm{CsI}(\mathrm{Tl})$ crystals that are used for WIMP search experiments performed at the Yangyang Underground Laboratory [15, 16, 17]. Each of the small-sized crystal was grown in the same ingot with one of the full-sized crystals. Two 3-inch PMTs (D726UK, Electron Tubes) with green-extended $\mathrm{RbCs}$ photocathodes were attached to each end of the CsI(Tl) crystal. The other four crystal surfaces were covered by two layers of Teflon $(0.2 \mathrm{~mm}$ thick) and black vinyl sheets. The amplified signals from the crystal and the neutron detectors were encoded by $400-\mathrm{MHz}$ flash analog-to-digital converters (FADCs) for a 32- $\mu$ s time interval. The CsI(Tl) crystals' photoelectron (PE) yields were measured to be $\approx 5 \mathrm{PE} / \mathrm{keV}$ using 59.54-keV $\gamma$-rays from an ${ }^{241} \mathrm{Am}$ calibration source. The trigger condition for the CsI(Tl) crystal was two or more PEs in each PMT within a 2- $\mu$ s time window, corresponding to a total of four or more PEs in the detector, which corresponds to a visible energy threshold of $\sim 1 \mathrm{keV}$. The event rate of the $\mathrm{CsI}(\mathrm{Tl})$ crystal during exposure to the neutron source was $\sim 300 \mathrm{~Hz}$, mostly due to background $\gamma$-rays produced in the source. This trigger rate was reduced to $\approx 0.3 \mathrm{~Hz}$ by requiring a time coincidence between the CsI(Tl) crystal and one of the neutron detectors within a $2 \mu$ s time window.

For the comparison with the electron recoil signals, the crystal was irradiated with $662-\mathrm{keV}$ $\gamma$-rays from a ${ }^{137} \mathrm{Cs}$ source that induced Compton scattering. These data were taken separately with the same setup except for a shield between the crystal and the Am-Be neutron source. The $\mathrm{CsI}(\mathrm{Tl})$ trigger condition was the same as that used for the neutron measurement, except for the 
time coincidence with one of the neutron detectors.

\section{Analysis}

\subsection{Neutron identification}

It is well known that BC501A liquid scintillator can separate neutrons from $\gamma$ rays using PSD [18, 19] because neutron-induced signals have a longer tail than $\gamma$-induced events with the same visible energy. Therefore, by using the ratio of total charge to the maximum pulse-height of the signal, neutrons are clearly separated from $\gamma$ rays, as can be seen in Fig. 2 (a).

The $2 \mu$ s coincidence between the CsI(Tl) crystal and one of the neutron detectors was contaminated by accidentals. This background was reduced by a stricter coincidence condition re-

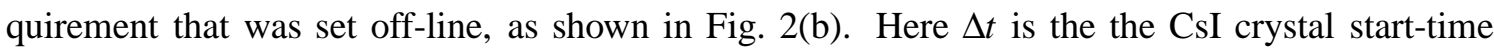
minus the start-time of the neutron detector. With good neutron separation capabilities and tighter time coincidence of the neutron detectors, we recorded neutron calibration data with the Am-Be neutron source over a two-month period for each crystal. Figure 3 shows the energy spectra of the neutron-induced events with the Am-Be source as well as the electron-induced events from ${ }^{137} \mathrm{Cs}$ source.

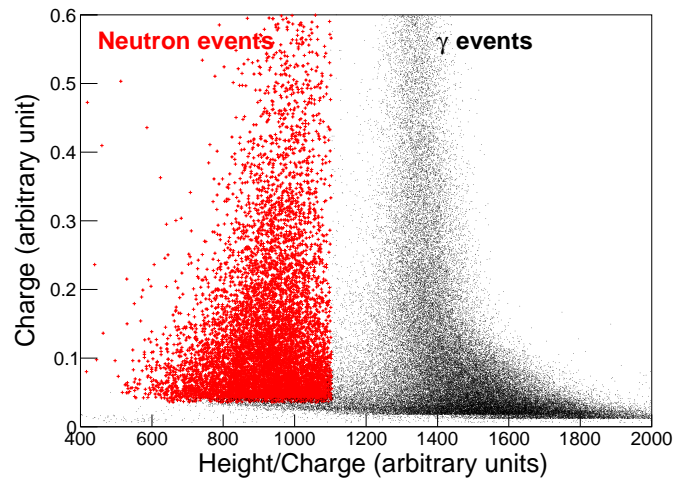

(a)

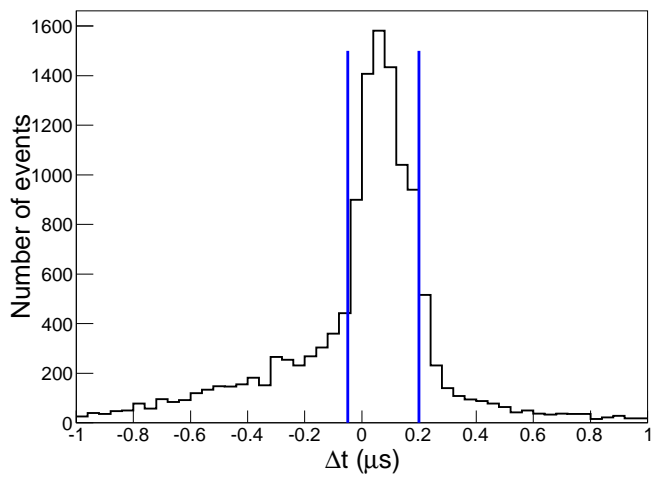

(b)

Figure 2. (a) The ratio of the maximun pulse height to the total charge (horizontal) versus the total charge for neutron-induced (solid red circles) and $\gamma$-induced (black points) for one of the neutron detectors. (b) Time difference between the $\mathrm{CsI}(\mathrm{Tl})$ crystal start-time and that of one of the neutron detectors.

\section{2 $\operatorname{CsI}(\mathrm{TI})$ data analysis}

Because the decay time of the scintillation light in the CsI(Tl) crystal is rather long, pulses from single photoelectrons are well separated in low visible-energy events. Single photoelectrons (SPEs) in an event were identified by the application of a clustering algorithm to the FADC data. From consecutive above-pedestal FADC bins, local maximum points are found and these are used to identify isolated clusters. In the case in which two local maxima are found in the same cluster of FADC bins, neighboring clusters are separated by locating the local minimum point between two clusters. When this technique is applied to ${ }^{55} \mathrm{Fe}$ source data, we find that in 5.9-keV signal events 


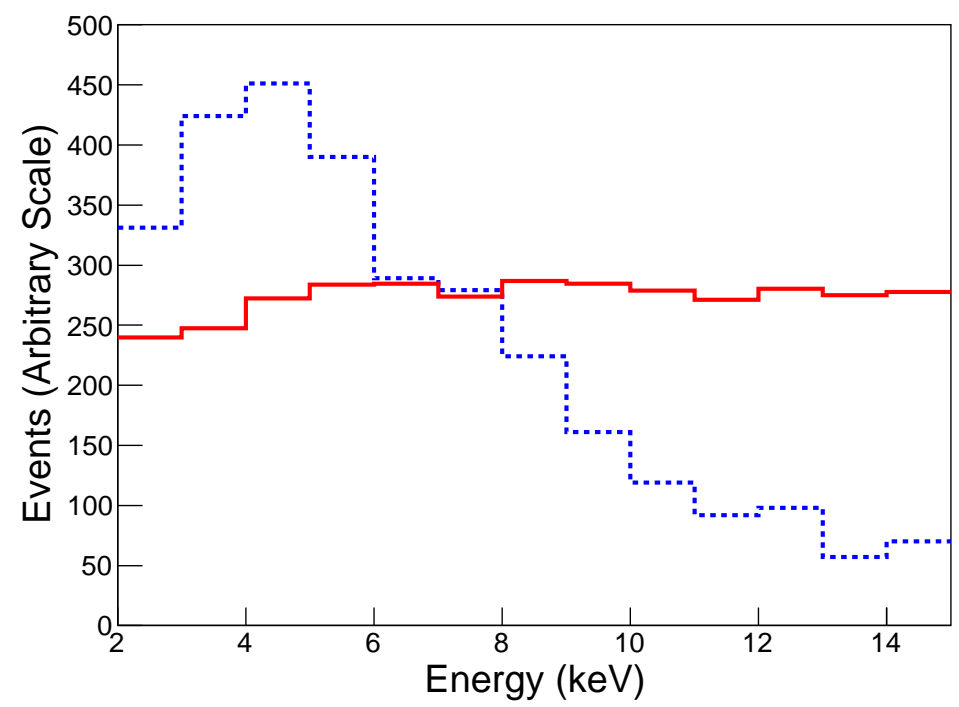

Figure 3. The low energy distribution of the neutron-induced events from the Am-Be neutron source (dashed line) and the electron-induced events from the ${ }^{137} \mathrm{Cs}$ source (solid line) are shown. The spectrum of electroninduced events is scaled down by a factor of 10 .

$>90 \%$ of the SPEs are well identified as isolated single clusters while the remaining SPEs are in overlapping clusters. A detailed explanation and an evaluation of the performance of the clustering algorithm can be found in Ref. [15].

The sum of the single-cluster charges over the entire time window is used to determine the deposited energy. Low-energy calibrations are done using the ${ }^{241} \mathrm{Am} 59.54-\mathrm{keV} \gamma$ peak. To characterize the pulse shapes of the nuclear- and electron-recoils, an unbinned maximum likelihood fit for the time distribution of photoelectrons in an event is performed with a double exponential function of the form:

$$
f(t)=\frac{1}{\tau_{f}} e^{-\left(t-t_{0}\right) / \tau_{f}}+\frac{R}{\tau_{s}} e^{-\left(t-t_{0}\right) / \tau_{s}},
$$

where $\tau_{f}$ and $\tau_{s}$ are decay time constants for the fast and slow components, respectively, $R$ is the ratio between the two components, and $t_{0}$ is the start time corresponding to the time of the first cluster in an event. Figure 4 shows an example of the fit result for a typical photoelectron time distribution of an event. We performed an event-by-event fit for both the neutron source data and the ${ }^{137} \mathrm{Cs}$ source data. From the fitted parameters, we calculated the mean time (MT) of each event as

$$
\mathrm{MT}=\int t \cdot f(t) d t / \int f(t) d t
$$

and used the MT as a PSD parameter to discriminate between nuclear- and electron-recoil events.

\subsection{Pulse shape discrimination}

It is possible to use PSD with the CsI(Tl) crystals because of the different time distributions of photoelectrons between nuclear- and electron-recoils [20]. We characterize the PSD power by a 


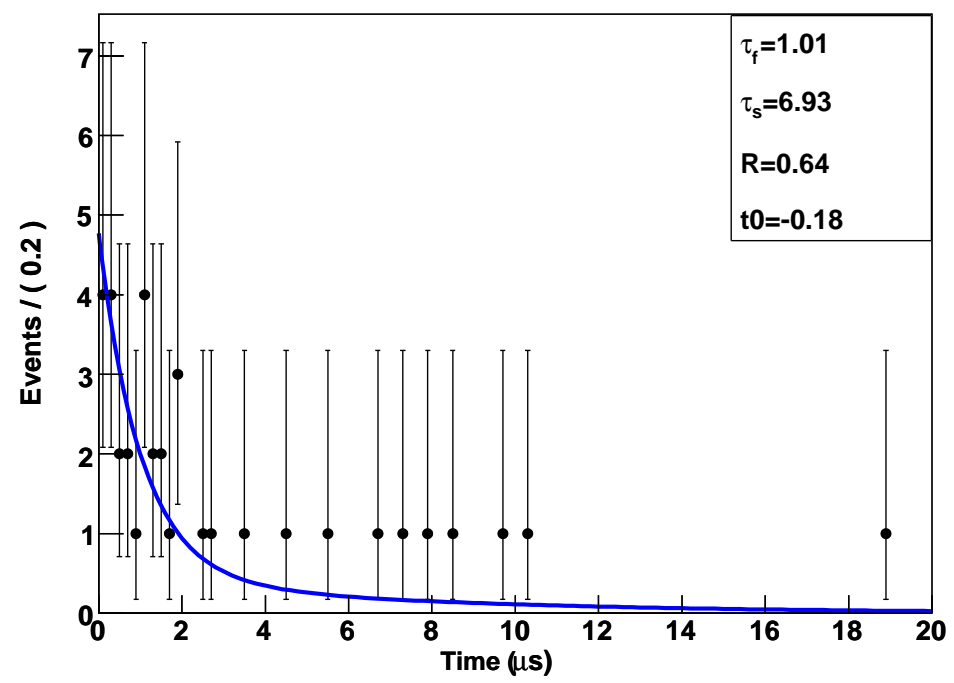

Figure 4. An example of the unbinned maximum likelihood fit of the time distribution of the identificed clusters using the two exponential decay components. This event corresponds to a neutron-induced event with a visible energy of $\sim 6-\mathrm{keV}$.

quality factor [21] defined as

$$
K \equiv \frac{\beta(1-\beta)}{(\alpha-\beta)^{2}}
$$

where $\alpha$ and $\beta$ are fractions of the nuclear- and electron-recoil events that satisfy the selection criteria, respectively, where we have varied these criteria to provide the best (i.e., the minimum) value of the quality factor. For a detector with perfect discrimination, $\alpha=1$ and $\beta=0$, thus a smaller quality factor means a better PSD power.

For a simple separation parameter, we choose the natural logarithm of MT $(\ln (\mathrm{MT}))$. Figure 5 shows the $\ln (\mathrm{MT})$ distributions for nuclear and electron recoil events in the Beijing crystal with visible energies in the range $5<E<6 \mathrm{keV}$. It is evident in this figure that the decay time for nuclear recoils is shorter than that for electron recoils, consistent with previous measurements [7], 8, 13, 14. We performed fits to the $\ln (\mathrm{MT})$ distributions using asymmetric Gaussian functions with $x=\ln (\mathrm{MT})$ as follows:

$$
\begin{aligned}
g(x)= & \frac{1}{1 / 2\left(\sigma_{L}+\sigma_{R}\right)} e^{-\frac{1}{2}\left(\frac{x-m}{\sigma_{L}}\right)^{2}}, x<m, \\
& \frac{1}{1 / 2\left(\sigma_{L}+\sigma_{R}\right)} e^{-\frac{1}{2}\left(\frac{x-m}{\sigma_{R}}\right)^{2}}, x \geq m,
\end{aligned}
$$

where $\sigma_{L}$ and $\sigma_{R}$ are standard deviations of left- and right-side Gaussians, respectively, and $m$ is the most probable value. This parameterization is slightly different from that in previous reports in which a simple Gaussian distribution was assumed [8, 14]. However, in this analysis we employed a different method to evaluate $\ln (\mathrm{MT})$ and used a much longer time window. Our data were well described by asymmetric Gaussians function, as can be seen in Fig. 5 . 


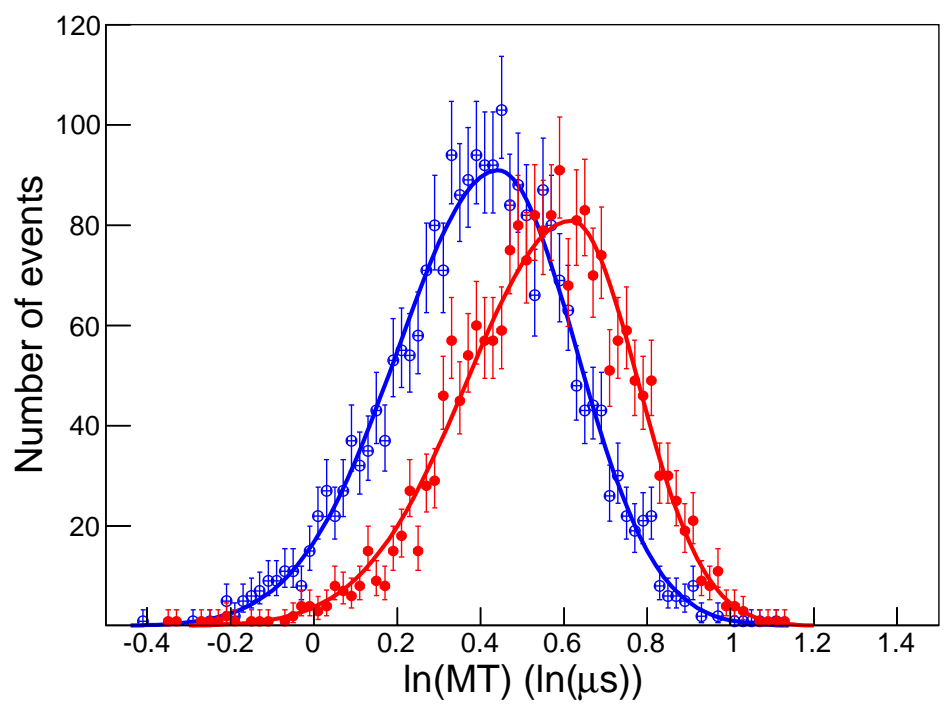

Figure 5. The $\ln (\mathrm{MT})$ distribution for the Beijing crystal's response to neutron-induced events (open circles) from the Am-Be source and electron-induced events (filled circles) from the ${ }^{137} \mathrm{Cs}$ source with visible energies between 5 and $6 \mathrm{keV}$. Superimposed are the results of a fit that uses asymmetric Gaussian functions.

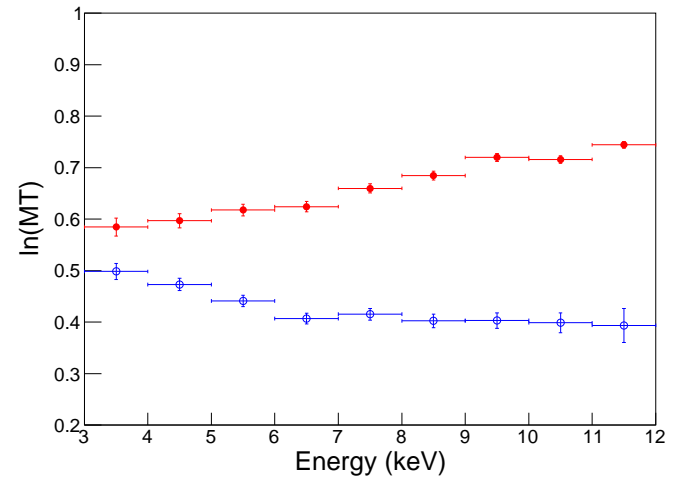

(a)

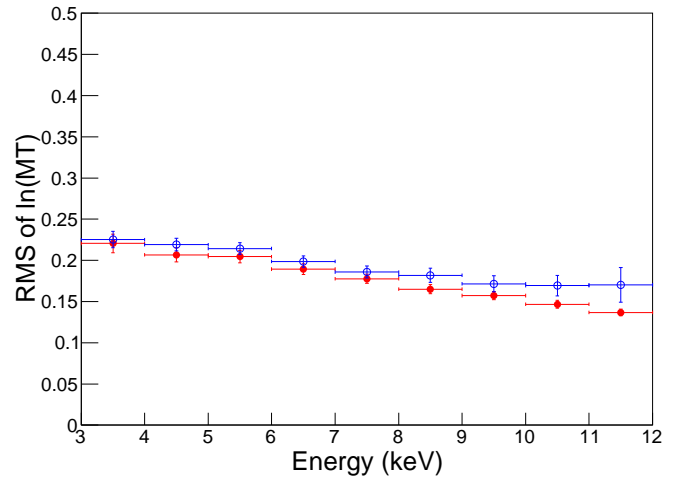

(b)

Figure 6. (a) The most probable value of $\ln (\mathrm{MT})$ from the asymmetric Gaussian fits for each energy bin for neutron-induced (open circles) and electron-induced (filled circles) events from the Am-Be source and the ${ }^{137} \mathrm{Cs}$ source, respectively, for the Beijing crystal. (b) The root mean square (RMS) of $\ln (\mathrm{MT})$ as a function of energy for neutron-induced (open circles) and electron-induced (filled circle) events.

We performed the fit for each $1-\mathrm{keV}$ energy bin from 3 to $12 \mathrm{keV}$ for both neutron and $\gamma$ calibration data. Figure 6 shows the most probable value and the root mean square (RMS) of $\ln (\mathrm{MT})$ from the asymmetric Gaussian fit of two different sets of calibration data. There is an energy dependence to the most probable values as well as the RMS values: at low energy, the separation power is reduced but we still retain some PSD capability at energies as low as $3 \mathrm{keV}$, a result that is similar to our previous measurement using the neutron generator [14].

To calculate the qaulity factors in Eq. 3.3, we provide simple selection criteria for $\ln (\mathrm{MT})$ as 


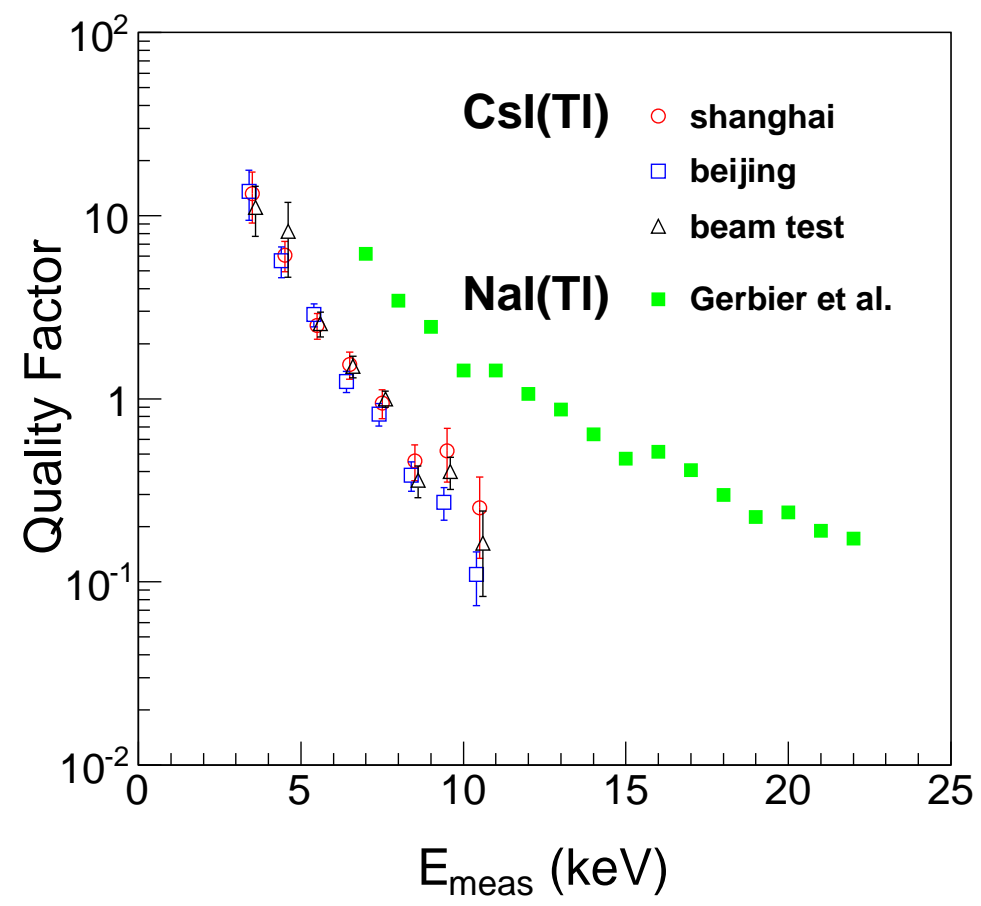

Figure 7. The extracted quality factors of the $\mathrm{CsI}(\mathrm{Tl})$ crystals compared to neutron generator data [14] and $\mathrm{NaI}(\mathrm{Tl})$ measurements [5].

$\ln (\mathrm{MT})<c$. We have varied these criteria $c$ in range between -0.5 and 1.5 to find the minimum value of the quality factor that maximizes the nuclear- to electron-recoil ratio in each keV bin events. Figure 7 shows the extracted (minimum) quality factors for the two CsI(Tl) crystals with the AmBe source compared with our previous result using the neutron generator as described in Ref. [14], where it is apparent that the quality factors obtained using the neutron source are consistent with those found using the neutron generator. This demonstrates that we can use the Am-Be neutron source for the PSD measurement of the $\mathrm{CsI}(\mathrm{Tl})$ crystal. We also find that the quality factors of the $\mathrm{CsI}(\mathrm{Tl})$ crystals are a factor of $\sim 10$ lower than those of $\mathrm{NaI}(\mathrm{Tl})$ crystals.

\section{Conclusions}

We constructed a neutron calibration facility using the recycled 300-mCi Am-Be source to study the response of $\mathrm{CsI}(\mathrm{Tl})$ crystals to nuclear recoil events. Even though this facility can not measure the quenching factor, we measure the PSD parameters between the nuclear recoil events and the electron recoil events of two CsI(Tl) crystals. The extracted quality factors are consistent with our previous measurements using the neutron generator. This gives us confidence in the use of this neutron calibration facility for the PSD measurements. The neutron calibration data of the two CsI(Tl) crystals obtained with this neutron calibration facility have already been used to extract the nuclear recoil events of WIMP search data obtained at the Yangyang Underground Laboratory [16, 
17, 22]. This neutron calibration facility is also being used to evaluate PSD capabilities of other candidate WIMP search scintillation detectors.

\section{Acknowledgments}

This research was funded by Grant No. IBS-R016-D1 and was supported by the Basic Science Research Program through the National Research Foundation of Korea (NRF) funded by the Ministry of Education (Grant No. NRF-2011-35B-C00007).

\section{References}

[1] B. W. Lee and S. Weinberg, Cosmological Lower Bound on Heavy-Neutrino Masses, Phys. Rev. Lett. 39 (1977) 165.

[2] G. Jungman, M. Kamionkowski, and K. Griest, Supersymmetric dark matter, Phys. Rep. 267 (1996) 195.

[3] R. J. Gaitskell, Direct Detection of Dark Matter, Annu. Rev. Nucl. Part. Sci. 54 (2004) 315; J. L. Feng and D. Sanford, Heart of darkness: the significance of the zeptobarn scale for neutralino direct detection, JCAP $\mathbf{0 5}$ (2011) 018 .

[4] J. B. Birks, Theory and Practice of Scintillating Counting, Pergamon, Oxford 1964.

[5] G. Gerbier et al., Pulse shape discrimination and dark matter search with NaI(Tl) scintillator, Astropart. Phys. 11 (1999) 287.

[6] B. Ahmed et al., The NAIAD experiment for WIMP searches at Boulby mine and recent results, Astropart. Phys. 19 (2003) 691.

[7] S. Pecourt et al., Calibration of a CsI(Tl) crystal with nuclear recoils and pulse shape measurements for dark matter detection, Astropart. Phys. 11 (1999) 457.

[8] V. A. Kudryavstev et al., CsI(Tl) for WIMP dark matter searches, Nucl. Instrum. Methods Phys. Res., Sect. A 456 (2001) 272.

[9] T. Fazzini et al., Pulse-shape discrimination with $\mathrm{CdWO} 4$ crystal scintillators, Nucl. Instrum. Methods Phys. Res., Sect. A 410 (1998) 213.

[10] L. Bardelli et al., Pulse-shape discrimination with $\mathrm{PbWO}_{4}$ crystal scintillators, Nucl. Instrum. Methods Phys. Res., Sect. A 584 (2008) 129.

[11] J. Glodo et al., Pulse Shape Discrimination with Selected Elpasolite Crystals, IEEE Trans. Nucl. Sci. 59 (2012) 2328.

[12] H. J. Kim et al., Test of CsI(Tl) crystals for the dark matter search, Nucl. Instrum. Methods Phys. Res., Sect. A 457 (2001) 471.

[13] S. C. Wu et al., Near threshold pulse shape discrimination techniques in scintillating CsI(Tl) crystals, Nucl. Instrum. Methods Phys. Res., Sect. A 523 (2004) 116.

[14] H. Park et al., Neutron beam test of CsI crystal for dark matter search, Nucl. Instrum. Methods Phys. Res., Sect. A 491 (2002) 460.

[15] H. S. Lee et al. (KIMS Collaboration), First limit on WIMP cross section with low background CsI(Tl) crystal detector, Phys. Lett. B 633 (2006) 201. 
[16] H. S. Lee et al. (KIMS Collaboration), Limits on Ineractions between Weakly Interacting Massive Particles and Nucleons Obtained with CsI(Tl) Crystal Detectors, Phys. Rev. Lett. 99 (2007) 091301.

[17] S. C. Kim et al. (KIMS Collaboration), New Limits on Interactions between Weakly Interacting Massive Particles and Nucleons Obtained with CsI(Tl) Crystal Detectors, Phys. Rev. Lett. 108 (2012) 181301.

[18] H. J. Kim et al., Measurement of the neutron flux in the CPL underground laboratory and simulation studies of neutron shielding for WIMP searches, Astropart. Phys. 20 (2004) 549.

[19] J. J. Zhu et al., Performance of a Large Volume Liquid Scintillation Detector for the Measurement of Fast Neutrons, J. Kor. Phys. Soc. 47 (2005) 202.

[20] G. F. Knoll, Radiation Detection and Measurements, John Wiley and Sons, 3rd. ed., New York 2000.

[21] R. J. Gaitskell et al., The Statistics of Background Rejection in Direct Detection Experiments for Dark Matter, Nucl. Phys. Proc. Suppl. B 51 (1996) 279.

[22] H. S. Lee et al. (KIMS Collaboration), Search for low-mass dark matter with CsI(Tl) crystal detectors, Phys. Rev. D 90 (2014) 052006. 